\section{Not just another primary care workforce crisis ...}

There is a baffling disconnect in the position of Irish and Purvis on the primary care workforce crisis. ${ }^{1}$ On the one hand, they say:

'The supply of newly qualified GPs is unlikely to match demand without international recruits and returners to the GP workforce.

On the other hand, many readers of this Journal will be astonished to learn the obstacles faced by UK-trained GPs who wish to return to England (but not Wales or Scotland, see below) after working as GPs for a period over 2 years in countries such as Australia, New Zealand, and Canada.

Briefly, they have to register for a local returners scheme, take a knowledge-based multiple choice question (MCQ) in London, wait for the results of that, then apply to do a basic objective structured clinical examination (OSCE) in London, wait for the results of that, then have a clinical interview with a regional educational supervisor and then, if all is deemed satisfactory, be signed off as fit to work, all the while idle at their own expense over a period of up to 6 months. This returners policy has been implemented by the Committee of General Practice Education Directors (COGPED), a body to that Irish and Purvis belong, with no attempt to distinguish at entry between a doctor who has been, say, on maternity leave and not working for 5 years and one who has been doing mainstream first world general practice in a comparable health economy. Arguments that the latter individual requires 'refamiliarisation' with the NHS are specious as no such 'refamiliarisation' is offered during the period they remain idle, their clinical skills atrophying. Further, knowledge of NHS procedures and protocols is not assessed by the MCQ and OSCE, which are basic clinical exams. Many would argue, too, that 'refamiliarisation' is not as complex a task as COGPED would have us believe and could easily be dealt with in many ways such as online learning modules or a short face-to-face course.

I suggest that Irish and Purvis reflect on the absurdity of COGPED's position and that if they are serious about tackling the workforce crisis they put in place a workable scheme for experienced UK-trained GPs returning from working in comparable health economies. Meanwhile, both Wales and Scotland take a far more pragmatic approach and will assess returning GPs on their merits via a clinical interview and do not require the $M C Q$ and $O S C E$, with the attendant period of costly, enforced idleness, as standard.

Either Irish, Purvis, and their colleagues on COGPED will put in place a more sensible regime to relicense UK GPs returning from abroad or we really will be in the workforce mire. In England, anyway.

\section{David Berger,}

MRCGP, Exmoor Medical Centre, Fishers Mead, Dulverton, Somerset, TA22 9EN.

E-mail: davebergerdamail.com

\section{Competing interests}

I may wish to work abroad and may wish to return one day. I have had previous correspondence on this issue with Irish and COGPED.

\section{REFERENCE}

1. Irish W, Purvis M. Not just another primary care workforce crisis ... Br J Gen Pract 2012; 62(597): 179-179.

DOI: 10.3399/bjgp12X641302

Irish and Purvis' have written a useful article summarising the imbalance between GPs entering and leaving the GP workforce, and indicate that this imbalance is likely to worsen in the next few years. The problems that the deaneries face now are acute and have serious implications for future recruitment of GPs at surgeries, and hence the viability of clinical services. There are two other dynamics in play that make the situation even more challenging than they describe.

First, many new roles are opening themselves up to GPs, and they currently sit somewhat uncomfortably alongside the traditional service roles of the general medical services and Personal Medical Services contracts. As a speciality we have accommodated training for many years. We have just about got enough appraisers. We have so far been able to recruit senior GPs to lead clinical commissioning groups. We have medical directors who are system leaders but nearly all of them are coping with too much work la lot of it protracted and complex) for the time they have available. All these additional roles are useful and interesting, and do contribute to patient care and safety. However, they all take GPs away from direct clinical work.

We have always seen some drift of GPs to post overseas, or moves sideways to other specialities such as occupational health.

So as a speciality we have many new roles opening up to all GPs, and we still have the patients to see. There may not be enough of us to go round all these roles. ${ }^{2}$

Secondly, we have a primary care service that is poorly configured in terms of its structures and processes to achieve the outcomes that both doctors and patients want and need. We have GPs working flat out in their surgeries coping with the daily treadmill of acute reactive demand. We know that there is much unmet need, but we feel so busy that meeting it can seem an impossible challenge. Our supposed 10minute consultations already average 11.7 minutes, and still fail to fully address all the problems patients have, and the comorbidity that needs addressing. We can see the challenges of age, complexity, and comorbidity are going to increase, and we are not well set up even for current demands. The GP's work is not well integrated with the specialist nurses available in primary care. Too often they are hospital outreach staff directed by consultants, rather than GPs. There are developing tools such as the Bolton Dashboard and the BUPA/Nuffield predictive risk management software that will in future allow us to ask 'who needs to be seen today?' as opposed to 'who's booked in today?'. But at present in our surgeries we are lumbered with the burden of acute reactive medicine and we struggle to see past our list of patients. And our work with our specialised nursing colleagues is not yet fully effective, and their work is not always best targeted.

So we see an ill-configured and specified primary care service with rising clinical and managerial demands on it, trying to meet it with too few staff. This scenario is 